\title{
Curative effect of surgical resection in the treatment of Kaposiform hemangioendothelioma
}

\author{
Song Zuo ${ }^{1}$, Xiaonan $\mathrm{Guo}^{1}$, Yanlin Wang ${ }^{1}$, Qiang Lou ${ }^{2}$, and Changxian Dong ${ }^{1}$ \\ ${ }^{1}$ Henan Provincial People's Hospital \\ ${ }^{2}$ Henan University
}

July 6,2020

\begin{abstract}
Background The clinical features and surgical outcomes of patients with Kaposiform hemangioendothelioma (KHE) is not fully clear. The purpose of this study is to better understand the therapeutic effect and hematological indices of surgical therapy for KHE. Procedure A retrospective study was conducted to review the medical documents of 43 patients with KHE who were treated with surgical resection at our hospital between February 2016 and November 2019. Multiple anatomical sites were involved. The curative effect was evaluated according to the tumor volume shrinkage, color shade, and blood examinations including platelet count, hemoglobin level, red blood cell number, clotting time and D-dimer concentration. Results For all cases underwent surgical treatment, the curative effects accounted for 100\%. At the end of the treatment, 9 children were followed up for 6 months, and no evidence of the recurrence of KHE was not found in any case. The number of red blood cells and clotting time were maintained at normal levels during different stage of treatment. The concentrations of hemoglobin and fibrinogen and the average number of platelets increased significantly after surgical treatment. The concentrations of D-dimer were much higher than normal value during admission with a high variability, and significantly decreased after surgery treatment. Further analysis found that the changing trends of D-dimer was positively correlated with fibrinogen protein or platelet number, but not clotting time. Conclusions The surgical treatment method is highly effective and of great significance for KHE therapy in pediatric clinic.
\end{abstract}

\section{Abstract \\ Background}

The clinical features and surgical outcomes of patients with Kaposiform hemangioendothelioma (KHE) is not fully clear. The purpose of this study is to better understand the therapeutic effect and hematological indices of surgical therapy for KHE.

\section{Procedure}

A retrospective study was conducted to review the medical documents of 43 patients with KHE who were treated with surgical resection at our hospital between February 2016 and November 2019. Multiple anatomical sites were involved. The curative effect was evaluated according to the tumor volume shrinkage, color shade, and blood examinations including platelet count, hemoglobin level, red blood cell number, clotting time and D-dimer concentration.

\section{Results}

For all cases underwent surgical treatment, the curative effects accounted for $100 \%$. At the end of the treatment, 9 children were followed up for 6 months, and no evidence of the recurrence of KHE was not found in any case. The number of red blood cells and clotting time were maintained at normal levels during different stage of treatment. The concentrations of hemoglobin and fibrinogen and the average 
number of platelets increased significantly after surgical treatment. The concentrations of D-dimer were much higher than normal value during admission with a high variability, and significantly decreased after surgery treatment. Further analysis found that the changing trends of D-dimer was positively correlated with fibrinogen protein or platelet number, but not clotting time.

\section{Conclusions}

The surgical treatment method is highly effective and of great significance for KHE therapy in pediatric clinic.

Key words: Kaposiform hemangioendothelioma, Kasabach-Merritt phenomenon, Long-term, Multifocal, Surgical treatment

\section{Introduction}

Kaposiform hemangioendothelioma (KHE) is a form of vascular tumor with poor prognosis, and is often hard to regress spontaneously. KHE affects approximately 0.91 in 100000 children ${ }^{1,2}$. According to the updated classification system issued by The International Society for the Study of Vascular Anomalies (ISSVA) in August $2018^{3}$, KHE is classified as aggressive but benign vascular tumor with a tendency to metastasize. The guidelines for KHE treatment is not available yet, and there are various treatment options including drugs (such as corticosteroids, vincristine, propranolol and interferon) intervention and surgical therapy ${ }^{4}$. Surgical resection is the optimal treatment for KHE among these treatment strategies; however, the clinical features and surgical outcomes of patients with KHE is not fully understood.

More than $70 \%$ patients with KHE develop a life-threatening complication called Kasabach-Merritt phenomenon (KMP) ${ }^{2,5}$, which was characterized by blood coagulation disorder and thrombocytopenia ${ }^{6}$. The thrombocytopenia is usually severe, with platelet counts of $21 \times 10^{9} / \mathrm{L}^{7}$ or $4 \times 10^{9} / \mathrm{L}^{8}$ at the initial presentation of KMP. Moreover, Elevated D-dimer and reduced fibrinogen levels in blood were often observed during or even before KMP onset ${ }^{9}$. D-dimer is degraded product of fibrin, and is formed by cross-linking of two D-domain fibrin monomers. The D-dimer level is usually undetectable under normal condition; however, the level of D-dimer will be elevated during clot formation. In spite of this, the changing trend of D-dimer during the occurrence of KMP and its correlations with other coagulation indexes such as fibrinogen level, platelet count and clotting time are still unclear.

KHE is found in varied locations, and the tumor is commonly localized in extremities and trunk ${ }^{10}$. Several vascular lesions such as Kaposiform lymphangiomatosis (KLA), infantile haemangioma (IH) resemble KHE clinically ${ }^{11}$, and may be localized in the mediastinum, lung, and thorax, etc. ${ }^{12}$. Thus, investigate the occurring rule of predilection sites might be beneficial for the KHE diagnosis.

In order to evaluate the curative effect of surgical resection in the treatment of KHE, the basic characteristics of patients with KHE from February 2016 to November 2019 in our hospital and hematological factors are discussed in this study. Moreover, the relationship between D-dimer and KHE, and the long-term outcome of surgical treatment of KHE were systematically assessed.

\section{Materials and methods}

General data. Forty-three children with KHEs treated in Henan Renmin hospital from February 2016 to November 2019 were selected, including 26 males and 17 females aged 5 days to 2 years. All children were diagnosed as KHEs. Inclusion criteria: 1) Kaposiform Hemangioendotheliomas with or without KasabachMerritt Phenomenon; 2) the age range is 0 - 12 years; 3) no other treatment option was given to these patients before; 4) children with no other tumor or hematological disease, neurological disease hepatic and renal dysfunction, diabetes, hypertension, cardiopulmonary insufficiency, tuberculosis, Epstein-Barr virus or cytomegalovirus infection before the treatment; 5) informed consents were signed by the children's guardians before the treatment. This study was approved by the medical Ethics Committee of Renmin Hospital of Henan province. 
Pre-treatment examinations. Blood routine examinations were done and hepatic and renal functions were detected before surgical treatment. In addition, the tumor size, location, texture and color were evaluated before and after treatment.

Treatment methods. Oral methylprednisolone therapy $(2 \mathrm{mg} / \mathrm{kg} /$ day $)$ was performed after admission to hospital. Platelets (1 unit) were transfused to those infants with low platelet counts $\left(<5 \times 10^{9} / \mathrm{L}\right)$ at 1 day before surgery. Tumors were completely resected and the curative effects and incidence of side effects were evaluated.

Curative effects evaluation. The curative effect was evaluated according to the tumor volume shrinkage, color shade, and blood examinations including platelet count, hemoglobin level, red blood cell number, clotting time, D-dimer concentration, etc. In addition, 9 children were followed up for 6 months at the end of the treatment.

Statistical analysis. The hematological data were expressed as mean $\pm \mathrm{SD}$, and were statistically analyzed with GraphPad prism software version 7.0. Intergroup comparisons were detected using the $t$-test. Pearson $\mathrm{r}$ correlation was used to measure the degree of the relationship between two variables. $\mathrm{P}<0.05$ represents that the difference was statistically significant.

\section{Results}

Basic characteristics of patients with KHE. The preoperative signs and symptoms in patients with KMP were summarized in Table 1. Most patients' age were less than 6 months. All patients had symptoms such as palpable lump and high skin temperature, and most patients had symptoms include local tenderness (90.2\%), obscure boundary (81.4\%), hemorrhage (46.5\%), deformity $(7 \%)$ and anabrosis $(2.3 \%)$. No bruising sign was observed in all patients. The tumor size with grade III and the tumor with purple red retained a majority of $44.2 \%$ and $65 \%$. Furthermore, the anatomical sites of the primary lesions in patients with KMP were summarized in Table 2. The left thigh, neck and right face were the most predilection sites of KHE.

Comparisons of curative effects on children. The enormous tumors in left upper arm, chest wall, and left cheek were all regressed after surgical treatment. For the 43 cases underwent surgical treatment, the curative effects accounted for $100 \%$. The changes in the tumor characteristics before (Fig.1A) and after (Fig. 1B) treatment were recorded. After treatment, the degree of surface tension was decreased, the tumor color turned pale red from dark red and the skin gradually returned to normal. At the end of the treatment, 9 children were followed up for 6 months, and no evidence of the recurrence of KHE was not found in any case.

Comparisons of hematological indexes during the treatment of KHE. Although obvious tumor regression after surgical treatment was observed, there may be difference in the recovery of blood clotting ability in these patients. Therefore, at the time of on admission, 0, 1, 2, 3, 5 days after surgery, before discharge, and half a year after discharge, the hematological indexes such as number of red blood cells and platelets, the concentrations of hemoglobin, fibrinogen and D-dimer, and clotting time were further examined. The number of red blood cells was maintained at normal levels during different stage of treatment (Fig. 2A). The concentrations of hemoglobin were slightly lower than normal level $(110.7 \mathrm{~g} / \mathrm{L})$ during admission, and increased significantly after surgical treatment, with an average level of $124.3 \mathrm{~g} / \mathrm{L}$ (Fig. 2B). The average number of platelets was at the lower limit of normal level during admission, and was recovered to $175.8 \times$ $10^{9} / \mathrm{L}$ immediately after surgery and continued to maintain at high levels even at half a year after surgery (Fig.2C). The clotting time were all at normal range during different stage of treatment (Fig. 2D). The concentrations of fibrinogen were slightly lower than normal level $(1.7 \mathrm{~g} / \mathrm{L})$ during admission, and increased significantly at 1 day after surgical treatment, with an average level of $2.7 \mathrm{~g} / \mathrm{L}$ (Fig. 2E). The concentrations of D-dimer were much higher than normal value during admission $(14.5 \mathrm{mg} / \mathrm{L})$, although with a high variability. Significantly decreased D-dimer values were observed after surgery treatment.

The Correlation Between D-dimer and other coagulation indexes in hemangioma. Due to considerable variability of D-dimer at different treatment stage of KHE, the level of D-dimer and other coagulation indexes such as fibrinogen concentration, the number of platelet and clotting time in serum were compared, and 
Pearson's correlation was used for analysis. The changing trends of D-dimer was positively correlated with fibrinogen protein or platelet number $(\mathrm{p}<0.05)$, but not clotting time $(\mathrm{p}>0.05)$ (Figure 3$)$.

\section{Discussion}

All infants were diagnosed as KME with thrombocytopenia, low fibrinogen and high D-dimer levels, and subsequently underwent surgical resection. Postoperative general observation showed the tumors were removed completely, and the skin color returned to normal. The postoperative hematological examination indicated the platelets count and coagulation profiles including thrombocytopenia, fibrinogen and D-dimer levels were restored

The KHE tumor often appears in the first 6 months of age, and occurs is found in varied locations and most commonly in thigh, neck and face, which is consistent with previous report ${ }^{7}$. The KHE tumor shows different responses from variable treatment strategies. Surgical resection is the optimal treatment for KHE among these treatment strategies, in this study, the comparison of the clinical curative effect before and after operation revealed that the clinical curative effect of surgical treatment was great; however, surgery may cause excessive bleeding due to thrombocytopenia, we transfused platelets to those infants with low platelet counts before surgery, which showed remarkable preventive effects on hemorrhage. In addition, the drug therapy for KHE using angiogenesis inhibitor sirolimus targeting the mammalian target of rapamycin (mTOR $)^{13-15}$, have been shown to be efficacious. While prednisolone, vincristine and sirolimus showed some effectiveness in decreasing the tumor size ${ }^{16,17}$, can cause side effects such as abdominal pain, elevations of aspartate aminotransferase (AST) and alanine aminotransferase (ALT), opportunistic infection, loss of appetite, etc 18. Moreover, there are case reports of visceral KHE not responding to sirolimus ${ }^{19,20}$. In addition, for those KHE patients unresponsive to glucocorticoids, propranolol, vincristine or sirolimus treatment, surgical therapy reduced skin petechia and coagulopathy to normal levels. It is worth noting these patients all didn't relapse. Thus, combination therapy may be a more efficacious treatment option in severe KHE especially in treating KHE.

The concentrations of D-dimer were much higher than normal value during admission $(14.5 \mathrm{mg} / \mathrm{L})$ (normal $<1 \mathrm{mg} / \mathrm{L}$ ), and remained elevated after the platelet count had returned to normal ${ }^{9}$. Although there was considerable variability of D-dimer at different treatment stage of KHE, D-dimer was significantly correlated with fibrinogen protein or platelet number, but not clotting time. Moreover, elevated D-dimer level is reported to be specific for various venous malformations, and is helpful in differentiating glomuvenous malformation with normal D-dimer level from other multifocal venous lesions ${ }^{21}$. In sum, D-dimer is a valuable factor and may be potential biomarker in evaluating surgical therapeutic effects of KHE.

The KMP is mainly characterized with thrombocytopenia and consumptive coagulopathy. Severe thrombopenia due to KMP may also make surgery difficult. Surgical treatment of the tumor might potentially worsen the thrombocytopenia and increase the hemorrhage risk ${ }^{22}$. However, in our study, the average number of platelets was recovered thoroughly and immediately after surgery and continued to maintain at high levels even at half a year after surgery, indicating that surgical treatment can efficiently alleviate thrombocytopenia and reduce the risk of bleeding.

In summary, the surgical therapy of KHEs can completely remove the tumor and significantly restore the skin color and texture. The surgical treatment method is highly effective and of great significance for KHE therapy in pediatric clinic.

\section{References}

1. Tower RL. Kaposiform haemangioendothelioma: new insights and old problems. The British journal of dermatology.2018;179(2):253-254.

2. Croteau SE, Liang MG, Kozakewich HP, et al. Kaposiform hemangioendothelioma: atypical features and risks of Kasabach-Merritt phenomenon in 107 referrals. J Pediatr. 2013;162(1):142-147. 
3. ISSVA Classification of Vascular Anomalies (C)2018 International Society for the Study of Vascular Anomalies Available at "issva.org/classification" Accessed 28.01.2020.

4. Ji Y, Chen S, Yang K, Xia C, Li L. Kaposiform hemangioendothelioma: current knowledge and future perspectives. Orphanet J Rare Dis.2020;15(1):39.

5. Zhang G, Gao Y, Liu X. Kaposiform haemangioendothelioma in a nine-year-old boy with Kasabach-Merritt phenomenon. Br J Haematol. 2017;179(1):9.

6. Mahajan P, Margolin J, Iacobas I. Kasabach-Merritt Phenomenon: Classic Presentation and Management Options. Clin Med Insights Blood Disord. 2017;10:1179545X17699849.

7. Ji Y, Yang K, Peng S, et al. Kaposiform haemangioendothelioma: clinical features, complications and risk factors for Kasabach-Merritt phenomenon. The British journal of dermatology.2018;179(2):457-463.

8. O'Rafferty C, O'Regan GM, Irvine AD, Smith OP. Kasabach-Merritt syndrome, kaposiform haemangioendothelioma and platelet blockade.Br J Haematol. 2015;171(1):11.

9. Boccara O, Fraitag S, Lasne D, et al. Kaposiform Haemangioendothelioma-spectrum Lesions with Kasabach-Merritt Phenomenon: Retrospective Analysis and Long-term Outcome. Acta Derm Venereol.2016;96(1):77-81.

10. Ji Y, Chen S, Li L, et al. Kaposiform hemangioendothelioma without cutaneous involvement. J Cancer Res Clin Oncol.2018;144(12):2475-2484.

11. Putra J, Gupta A. Kaposiform haemangioendothelioma: a review with emphasis on histological differential diagnosis. Pathology.2017;49(4):356-362.

12. Ji Y, Chen S, Peng S, Xia C, Li L. Kaposiform lymphangiomatosis and kaposiform hemangioendothelioma: similarities and differences. Orphanet J Rare Dis. 2019;14(1):165.

13. Wang Z, Zheng C, Sun H, et al. Immunohistochemical Analysis of mTOR Pathway-Related Proteins in Kaposiform Hemangioendothelioma.Dermatology. 2020:1-9.

14. Chinello M, Di Carlo D, Olivieri F, et al. Successful Management of Kaposiform Hemangioendothelioma with Long-Term Sirolimus Treatment: a Case Report and Review of the Literature. Mediterr J Hematol Infect Dis. 2018;10(1):e2018043.

15. Matsumoto H, Ozeki M, Hori T, et al. Successful Everolimus Treatment of Kaposiform Hemangioendothelioma With Kasabach-Merritt Phenomenon: Clinical Efficacy and Adverse Effects of mTOR Inhibitor Therapy. J Pediatr Hematol Oncol. 2016;38(8):e322-e325.

16. Zhang G, Chen H, Gao Y, Liu Y, Wang J, Liu XY. Sirolimus for treatment of Kaposiform haemangioendothelioma with Kasabach-Merritt phenomenon: a retrospective cohort study. The British journal of dermatology. 2018;178(5):1213-1214.

17. Cashell J, Smink GM, Helm K, Xavier F. Kaposiform hemangioendothelioma with Kasabach-Merritt phenomenon in an infant: Successful treatment with prednisolone, vincristine, and addition of sirolimus. Pediatr Blood Cancer. 2018;65(12):e27305.

18. Peng S, Yang K, Xu Z, Chen S, Ji Y. Vincristine and sirolimus in the treatment of kaposiform haemangioendothelioma. J Paediatr Child Health. 2019;55(9):1119-1124.

19. Triana PJ, Dore M, Nunez VC, et al. Pancreatic Kaposiform Hemangioendothelioma Not Responding to Sirolimus. European J Pediatr Surg Rep. 2017;5(1):e32-e35.

20. Wu Y, Qiu R, Zeng L, et al. Effective surgical treatment of life-threatening huge vascular anomalies associated with thrombocytopenia and coagulopathy in infants unresponsive to drug therapy. BMC Pediatr. 2020;20(1):187. 
21. Dompmartin A, Ballieux F, Thibon P, et al. Elevated D-dimer level in the differential diagnosis of venous malformations. Arch Dermatol. 2009;145(11):1239-1244.

22. Wong BL, Lee VN, Tikka T, Kim D, Dwivedi RC. Kaposiform haemangioendothelioma of the head and neck. Crit Rev Oncol Hematol. 2016;104:156-168.

\section{Figure legends:}

Figure 1. Changes in the tumor characteristics: (A) before treatment; (B) after treatment.

Figure 2. Hematological indexes before and after the treatment of KHE. At the time of on admission (n $=43), 0,1,2,3,5$ days after surgery $(\mathrm{n}=43)$, before discharge $(\mathrm{n}=43)$, and half a year after discharge $(\mathrm{n}=9)$, the hematological indexes such as number of red blood cells and platelets, the concentrations of hemoglobin, fibrinogen, D-dimer and clotting time were examined.

Figure 3. The correlation between D-dimer and fibrinogen or platelet number or clotting time in hemangioma. Pearson $\mathrm{r}$ correlation was used to measure the degree of the relationship between two variables. $\mathrm{P}<0.05$ represents that the difference was statistically significant.

\section{Statement of Ethics}

The study was approved by the Ethics Committee of the Renmin Hospital of Henan province, and written informed consent was obtained from the parents of each patient.

\section{Acknowledgements}

Not applicable.

\section{Competing interests}

The authors declare that there are no conflicts of interest in the present study.

\section{Funding}

This study was funded by the National Natural Science Foundation of China (grant no. 81970592), Key Scientific and Technological Project of Henan Province (grant no. 192102310314 and 202102310380), Research Fund of Henan University and Research Program of Henan Provincial People's Hospital (grant no. SB201901070).

\section{Availability of data and materials}

The datasets used and/or analyzed during the present study are available from the corresponding author on reasonable request.

\section{Author Contributions}

SZ and QL conceived and designed the study. SZ collected the data. SZ, XG, YW, QL, and CD analyzed the data. SZ and QL edited and revised the manuscript. All authors approved the final version of the manuscript.

\section{Hosted file}

Tables.docx available at https://authorea.com/users/337792/articles/463359-curative-effectof-surgical-resection-in-the-treatment-of-kaposiform-hemangioendothelioma 


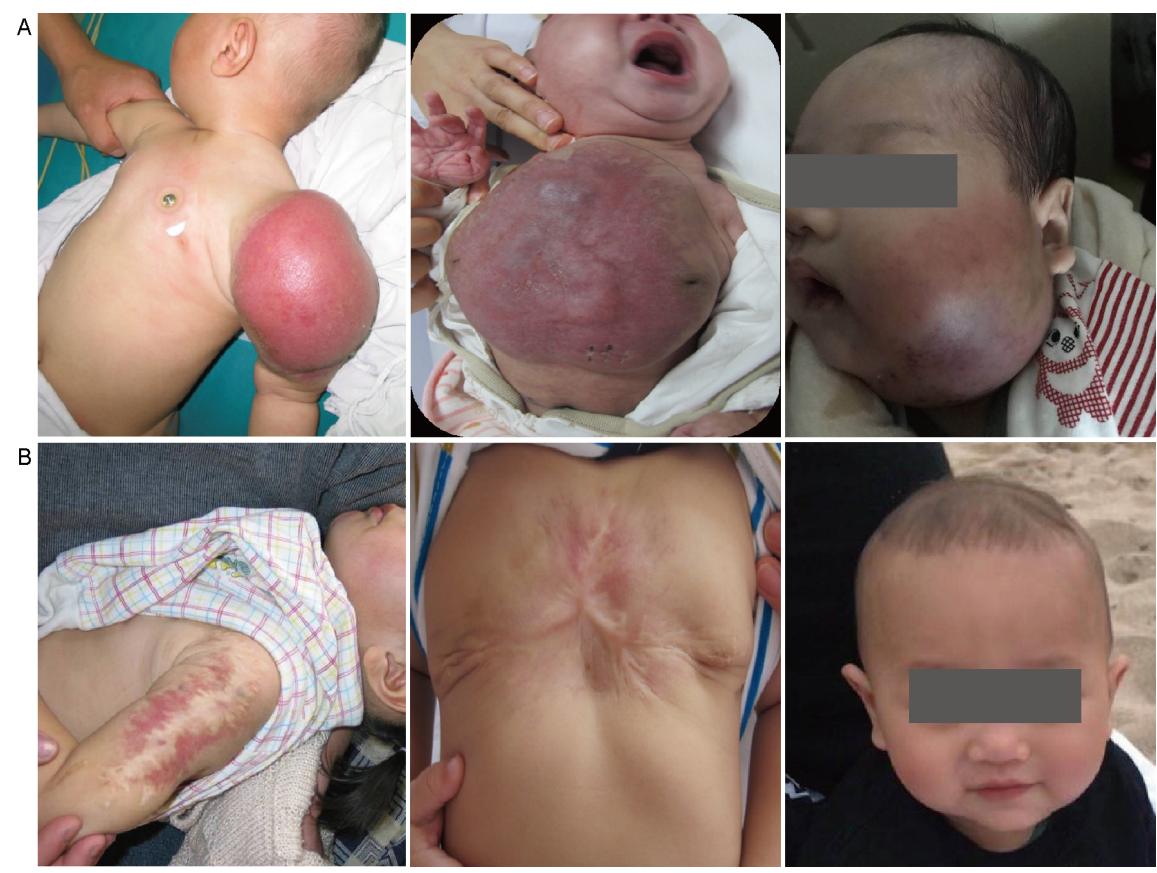

A
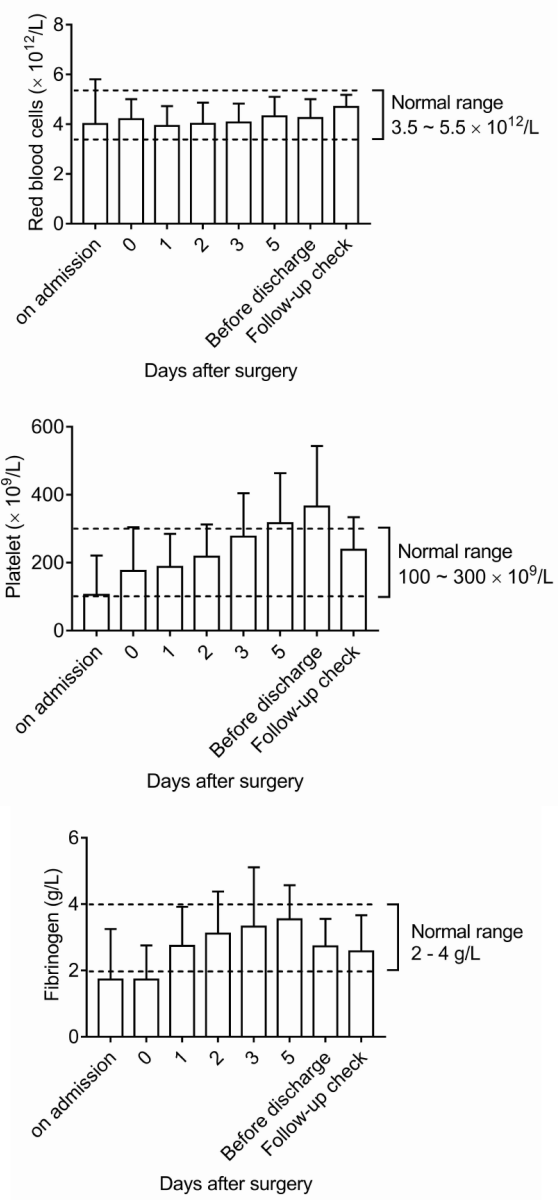

B

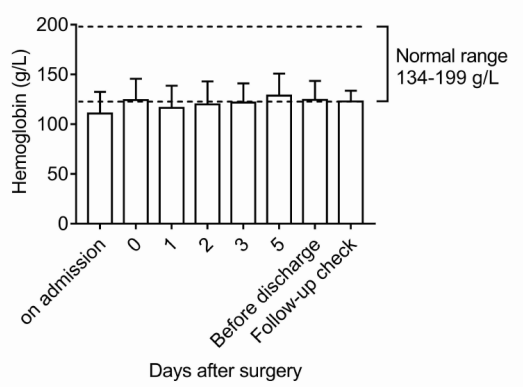

D

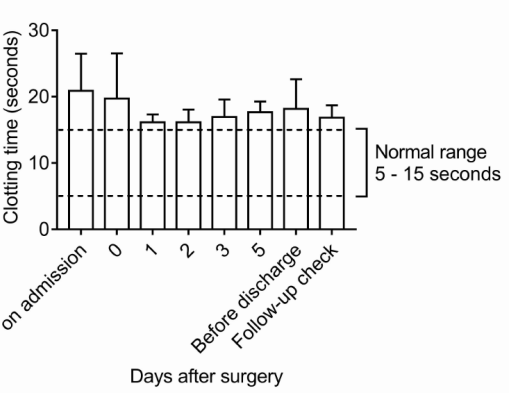

F

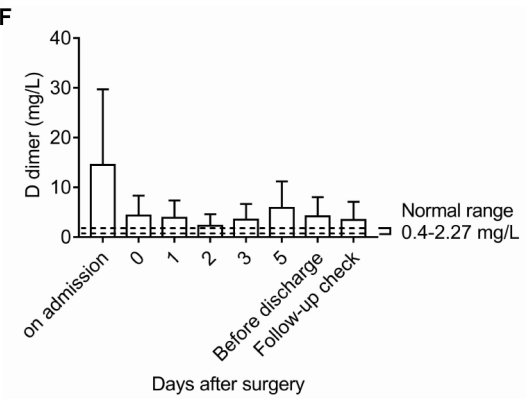


A

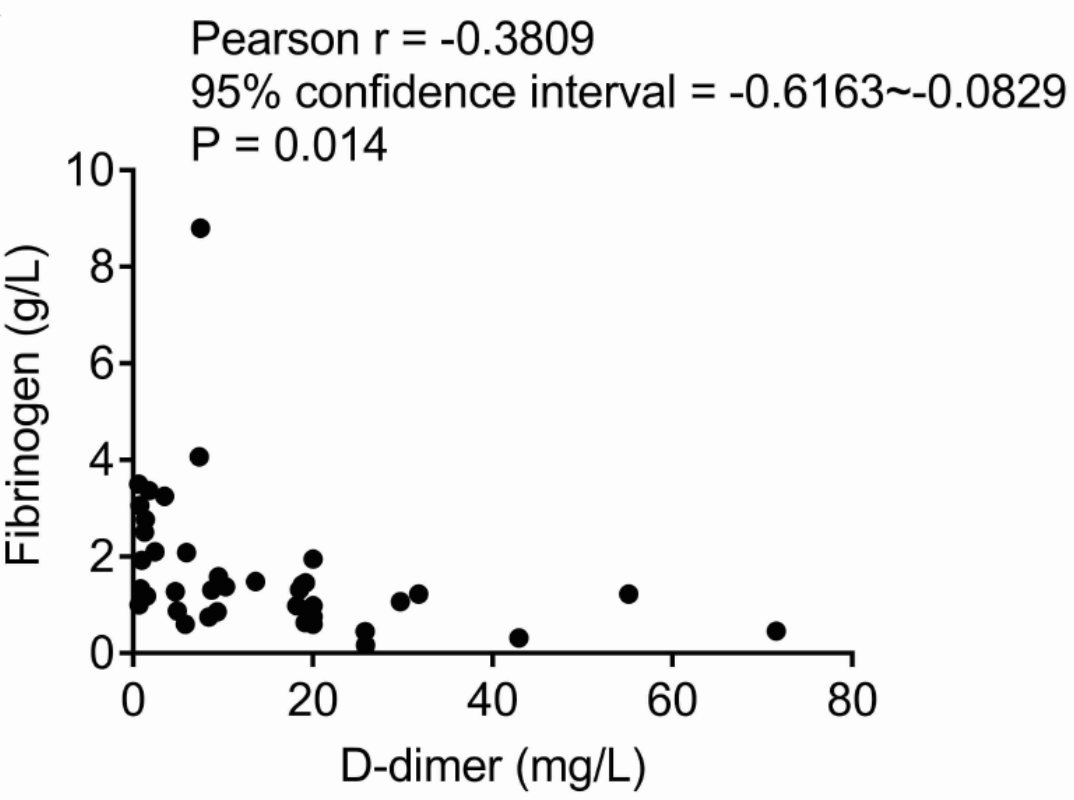

B

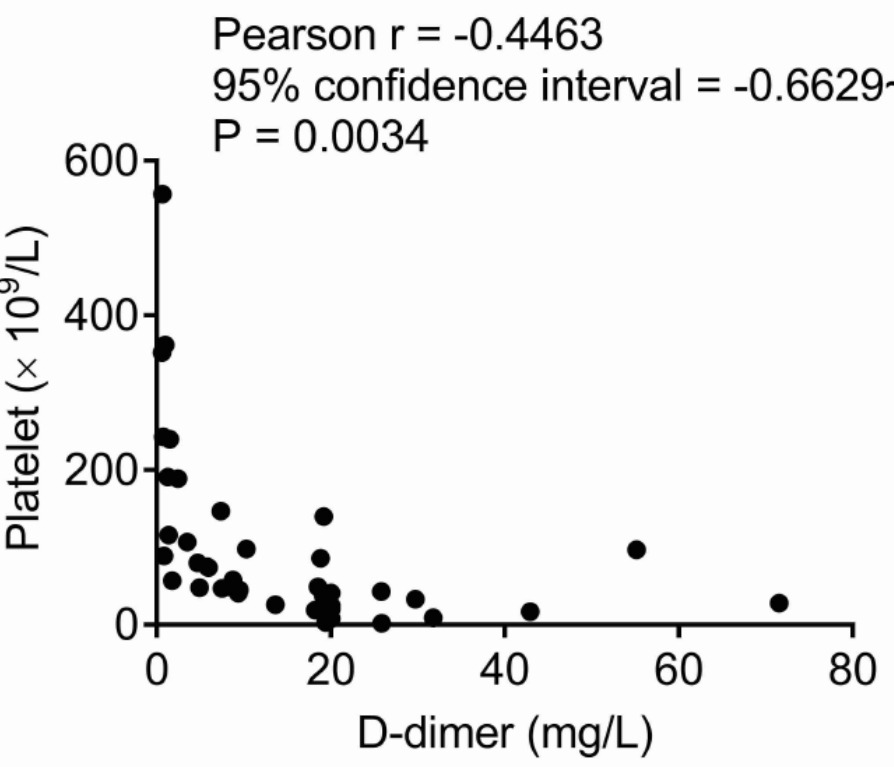

C

Pearson $r=0.2928$

$95 \%$ confidence interval $=-0.01634 \sim 0.5508$

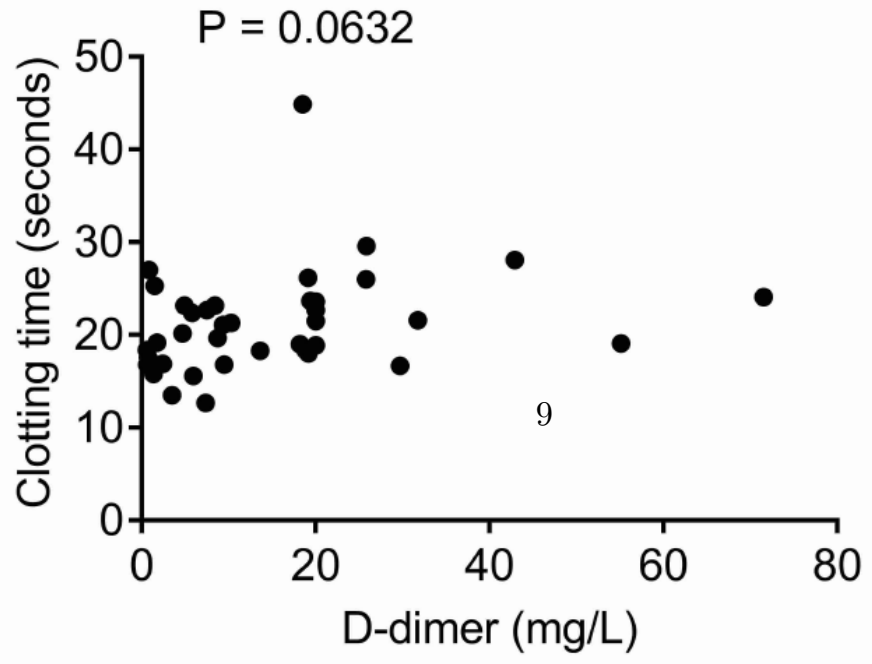

